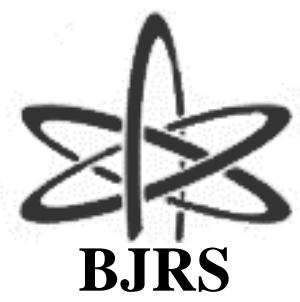

BRAZILIAN JOURNAL

$\mathrm{OF}$

RADIATION SCIENCES

08-03B (2021) 01-16

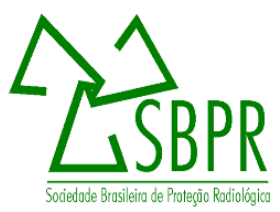

\title{
Two-dimensional transient thermal analysis of a fuel rod by finite volume method
}

\author{
R. Y. N. Costa ${ }^{\text {a }}$ M. A. B. Silva ${ }^{a}$; C. A. B. O. Lira \\ ${ }^{a}$ Universidade Federal de Pernambuco/ Departamento de Energia Nuclear, 50740-545, Recife, PE, Brazil \\ ryncosta@gmail.com; mabs500@gmail.com; cabol@ufpe.br
}

\begin{abstract}
One of the greatest concerns when studying a nuclear reactor is the warranty of safe temperature limits all over the system at all times. The preservation of core structure along with the constraint of radioactive material into a controlled system are the main focus during the operation of a reactor. The purpose of this paper is to present the temperature distribution for a nominal channel of the AP1000 reactor developed by Westinghouse Co. during steady-state and transient operations. In the analysis, the system was subjected to normal operation conditions and then to blockages of the coolant flow. The time necessary to achieve a new safe stationary stage (when it was possible) was presented. The methodology applied in this analysis was based on a two-dimensional survey accomplished by the application of Finite Volume Method (FVM). A steady solution is obtained and compared with an analytical approach that disregards axial heat transport to determine its relevance. The results show the importance of considering axial heat transport in this type of study. A transient analysis shows the behavior of the system when submitted to coolant blockage at channel's entrance. Three blockages were simulated $(10 \%$, $20 \%$ and $30 \%$ ) and the results show that, for a nominal channel, the system can still be considerate safe (there's no bubble formation until that point).
\end{abstract}

Keywords: Finite Volume Method, Thermal analysis, AP1000 nuclear reactor. 


\section{INTRODUCTION}

The AP1000 reactor is the APWR (Advanced Pressurized Water Reactor) developed by Westinghouse Company that adopts proved technology from 35 years of experience in the market along passive technology and modular construction to improve its performance [1]. All these characteristics make this reactor the safer and more economically competitive of all APWRs [2], resulting it one of the most researched reactors in use. [1] and [2] describe the design and economics involved in AP1000's project. In 2012, [3] developed the code FROBA (Fuel ROd Behavior Analysis) to predict the temperature distribution variation due to burnup effects.

AP1000 is a heterogenous reactor and the power generated in each point of the fuel rod is given by Equation (1) in the fuel material and is null in other regions [4]. In (1), $q_{0}{ }^{\prime \prime \prime}\left[\frac{\mathrm{w}}{\mathrm{m}^{3}}\right]$ is the maximum volumetric heat generation, $\kappa_{f}\left[m^{-1}\right]$ is the reciprocal of fuel diffusion length, $H[m]$ is rod's length, $r[m]$ and $z[m]$ are radial and axial position of the analyzed point related to the center of the rod $(r=0)$ and to the south extremity $(z=0)$.

$$
q^{\prime \prime \prime}=q_{0}{ }^{\prime \prime \prime} \cdot I_{0}\left(\kappa_{f} r\right) \cdot \sin \left(\frac{\pi z}{H}\right)
$$

Since the linear heat generation $\left(Q_{\text {linear }}\right)[W]$ is given by the integration of the volumetric heat generation times the rods' lateral area, the maximum volumetric heat generation is given by Equation (2), where $R_{f}[m]$ is the radius of the fuel cylinder.

$$
q_{0}^{\prime \prime \prime}=\frac{Q_{\text {linear }} \cdot \kappa_{f}}{4 R_{f} I_{1}\left(\kappa_{f} R_{f}\right)}
$$

To verify the safety in normal steady state and in transients related to coolant blockage at channel entrance, a two-dimensional thermal analysis in a nominal channel of AP1000 reactor is done. It is 
considered that the heat is transported through the materials within the rod by conduction in both radial and axial directions, and through convection in coolant only in axial direction due to its movement [5]. The coolant entrance temperature is known and always equal to $\mathrm{T}[\mathrm{K}]$.

By a one-dimensional analysis, [7] determined the temperature distribution of a homogenous cylinder through CATHENA software with FVM (Finite Volume Method) and FEM (Finite Elements Method). FVM delivers better results even with a less refined mesh, while FEM even produced an inconsistent result (increase in temperature when the system was submitted to cooling effects). In 2013, [8] presented a thermal analysis of a nuclear fuel rod without gap through FVM to evaluate flux depression factor, with more realistic results. The analysis is done through the FVM due to its capability of ensuring local and global conservation of properties and its adequacy to system with great symmetry [5].

A nominal channel from AP1000 reactor is analyzed. The channel contains a fuel rod composed by a uranium dioxide cylinder with a ZIRLO cladding, and a gap filled with helium. It is considered a perfect fuel rod, perfectly symmetrical and without fabrication issues. The temperature distribution in different scenarios is determined and discussed for each case analyzed through a comparison between a numerical analysis that considers the heat transmitted both radial and axially and ElWakil's [6] solutions that ignore the axial fraction of heat dispersion.

The numerical analysis of AP1000's nominal channel is accomplished through the division of the system into control volumes (CVs) and an individual thermal analysis for each group of control volumes that presents the same characteristics (physical and thermal). The channel is divided as shown in Figure 1, where $N_{z}$ are the number of axial divisions in the fuel rod, and $N_{f}, N_{g}$ and $N_{c}$ are the number of radial divisions in the fuel material, in gap region and in cladding, respectively. The rod is divided in seven regions: central CVs, fuel CVs, interface fuel-gap, gap CVs, interface gap-cladding, cladding CVs, interface cladding-coolant, according to its materials and border's differences, that would give different final coefficients for the numerical equations. The control volumes in the extremities of the rod are exposed to coolant's heat transfer influence, and their length is half of the length inside the fuel rod. In the coolant region, there are four sections: entrance chamber, exit chamber, CV immediately after the entrance chamber and CVs intern to the channel. 
The ideal mesh was decided after compiling a finer mesh $\left(N_{z}=1000, N_{f}=1000, N_{g}=100\right.$, $N_{c}=100$ ) and reducing it to a mesh that would give the same maximum temperature (with a $0.1 \%$ tolerance over the obtained through the finer mesh). The adopted mesh contains $N_{z}=50, N_{f}=$ $114, N_{g}=50, N_{c}=10$.

Figure 1: Scheme channel's $C V$ division $\left(N_{f}=3, N_{g}=2, N_{c}=3\right.$ e $\left.N_{z}=3\right)$.
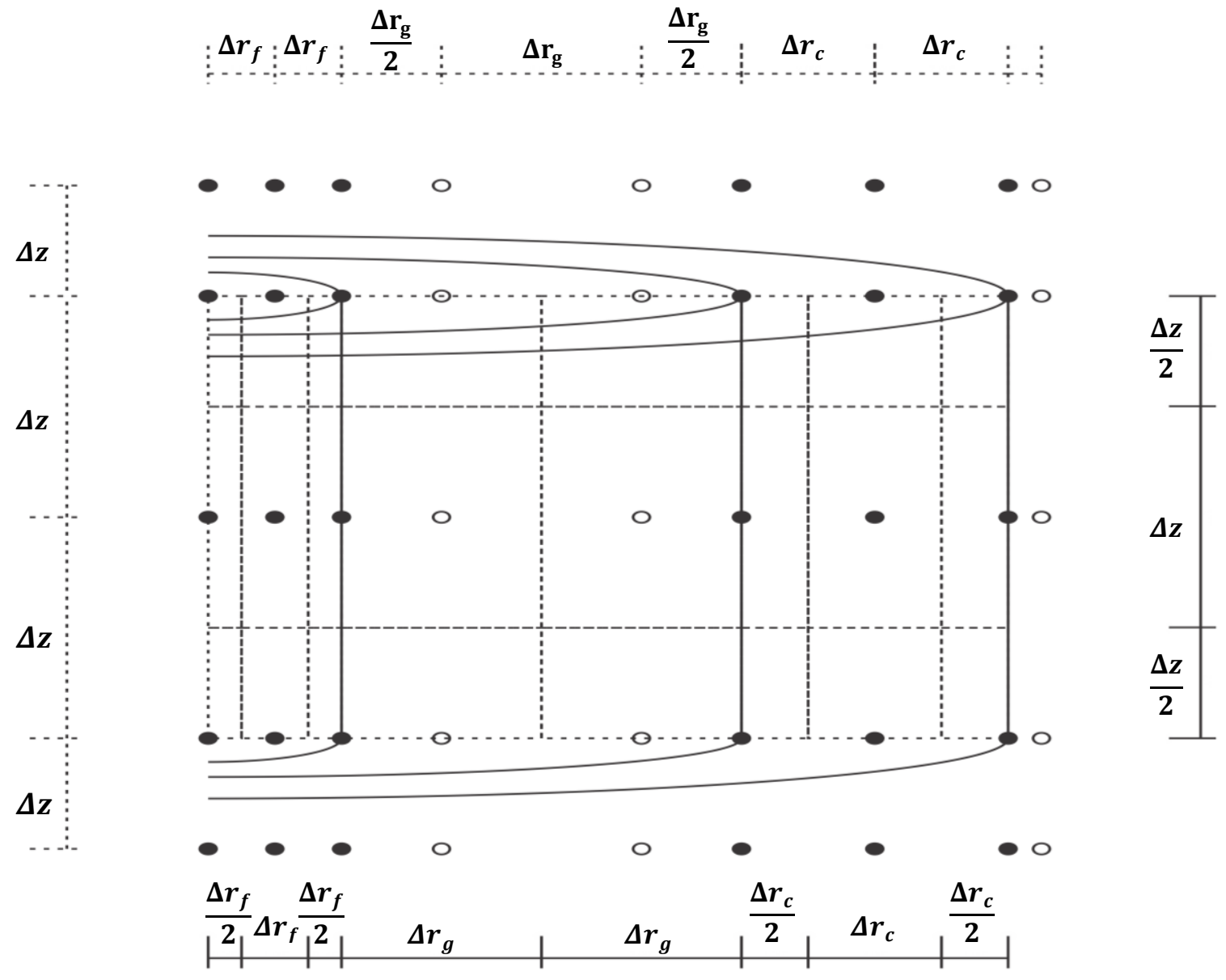

Applying energy balance in each control volume, the coefficients for the numerical solution can be found. In the fuel rod, the heat is transmitted by conduction through each material, including in the 
gap due to its small thickness [9]. In the coolant, the heat is transmitted primordially by convection in coolant's flow direction [5] and that's the only term acknowledge during this work. To apply the energy balance in each control volume, some material properties are necessary, and those vary with temperature. Then, uranium dioxide density can be calculated by correlations from [10], its conduction coefficient from [11] and its specific heat from [12]. For ZIRLO, the density was considered constant and equal to $6.57 \cdot 10^{3} \mathrm{~kg} / \mathrm{m}^{3}$ according to [13], and its conductivity and specific heat were determined by correlations given by [12]. The properties for helium and water were determined through REFerence fluid PROPerties (REFPROP) module from [14]. Finally, the convection coefficient for the coolant was determined from the correlation given by [11]. Other initial properties to allow the temperature determination were taken from [11].

\section{NUMERICAL SOLUTION (TRIDIAGONAL MATRIX ALGORITHM)}

Through the application of Energy Balance in each control volume group, the coefficients were determined and organized in matrix form, generating a pentadiagonal matrix for each axial position (the coefficients can be found in [15]). For each control volume group, the coefficients for the TriDiagonal Matrix Algorithm (TDMA) were determined (presented in [15]) and the system was solved through author's MatLab developed code. As suggested by [5], the method adopted to solve the matrix system was the TriDiagonal Matrix Algorithm (TDMA), that uses simple matrix inversion to determine the unknown terms of the problem as long as the coefficient matrix is a square tridiagonal inversive matrix. To transform the pentadiagonal coefficient matrix in a tridiagonal one, the temperatures for all radial positions in each axial position, from channel's entrance to its exit, is determined by assuming the north neighbor's temperature as equal to south's. Since the temperature at the channel's entrance is known, this is the south temperature for the first axial position. This being a known term, along with the north's temperature (supposed to be known), these terms must be moved to the results matrix (Matrix $B$ ). When this axial position is determined through matrix inversion ( $T=A^{-1} \cdot B$, through TDMA method), this will be south's temperature for the new axial position. This way, the south temperature is always known. Finally, this distribution obtained should not be the correct numerical solution since all north temperatures were assumed as equal to 
south temperatures; but those values can be used to recalculate the temperature distribution and to obtain a value closer to the correct numerical solution. This procedure must be repeated until there's convergence of the results between two simultaneous distributions (until their difference is no higher than $\left.10^{-3} \%\right)$. The last distribution obtained is the numerical solution for the case study.

\section{STEADY STATE ANALYSIS}

A nominal channel from AP1000 reactor is analyzed to determine the steady temperature distribution when it is operating at normal conditions. A numerical analysis through Finite Volume Method is done and compared to the temperature distribution of external cladding wall and coolant obtained by El-Wakil's equations.

\subsection{ANALYTICAL ANALYSIS}

Coolant's and outer wall's temperature distributions are determined through the analytical equations given by El-Wakil's [5] Equations (3) and (4), respectively.

$$
\begin{gathered}
T_{\text {coolant }}=T+\frac{q_{0}^{\prime \prime \prime} R_{f}^{2} H}{c_{p} \dot{m}}\left(\cos \left(\pi+\frac{\pi z}{H}\right)+1\right) \\
T_{\text {wall }}=T+q_{0}^{\prime \prime \prime}\left(\pi R_{\text {total }}^{2}\right)\left(\frac{H}{\pi c_{p} \dot{m}}\left(\cos \left(\pi+\frac{\pi z}{H}\right)+1\right)+\frac{\operatorname{sen}\left(\frac{\pi z}{H}\right)}{2 \pi h R_{\text {total }}}\right)
\end{gathered}
$$

At (3) and (4), $T[K]$ is the coolant temperature at channel's entrance, $c_{p}\left[\frac{\mathrm{J}}{\mathrm{kg} \cdot \mathrm{K}}\right], \dot{m}\left[\frac{\mathrm{kg}}{\mathrm{s}}\right]$ and $h\left[\frac{\mathrm{w}}{\mathrm{m}^{2} \cdot \mathrm{K}}\right]$ are the specific heat, mass flow and convection coefficient of water, respectively. $R_{\text {total }}[\mathrm{m}]$ is the fuel rod's total radius. 


\subsection{RESULTS}

The numerical solution was obtained by applying energy balance over each control volume to determine the temperature in the center of the control volume. The equation system was then solved through Finite Volume Method at MatLab software according to self-developed code. Finally, the temperature distribution for the rod's outer wall and coolant for the numerical solution and analytical solutions (through Equations (3) and (4)) were compared.

The numerical solution considered a mesh composed by $N_{z}=50, N_{f}=114, N_{g}=50, N_{c}=10$ and the solution was obtained in 6.775 seconds, after only 7 iterations. The maximum temperature obtained by the numerical analysis was $1482.21^{\circ} \mathrm{C}$. Figure 2 presents the numerical solution for the radial temperature distribution at nominal channel's medium height, showing each material's temperature profile.

Figure 2: 2D Steady state radial temperature distribution medium height in AP1000 nominal channel 


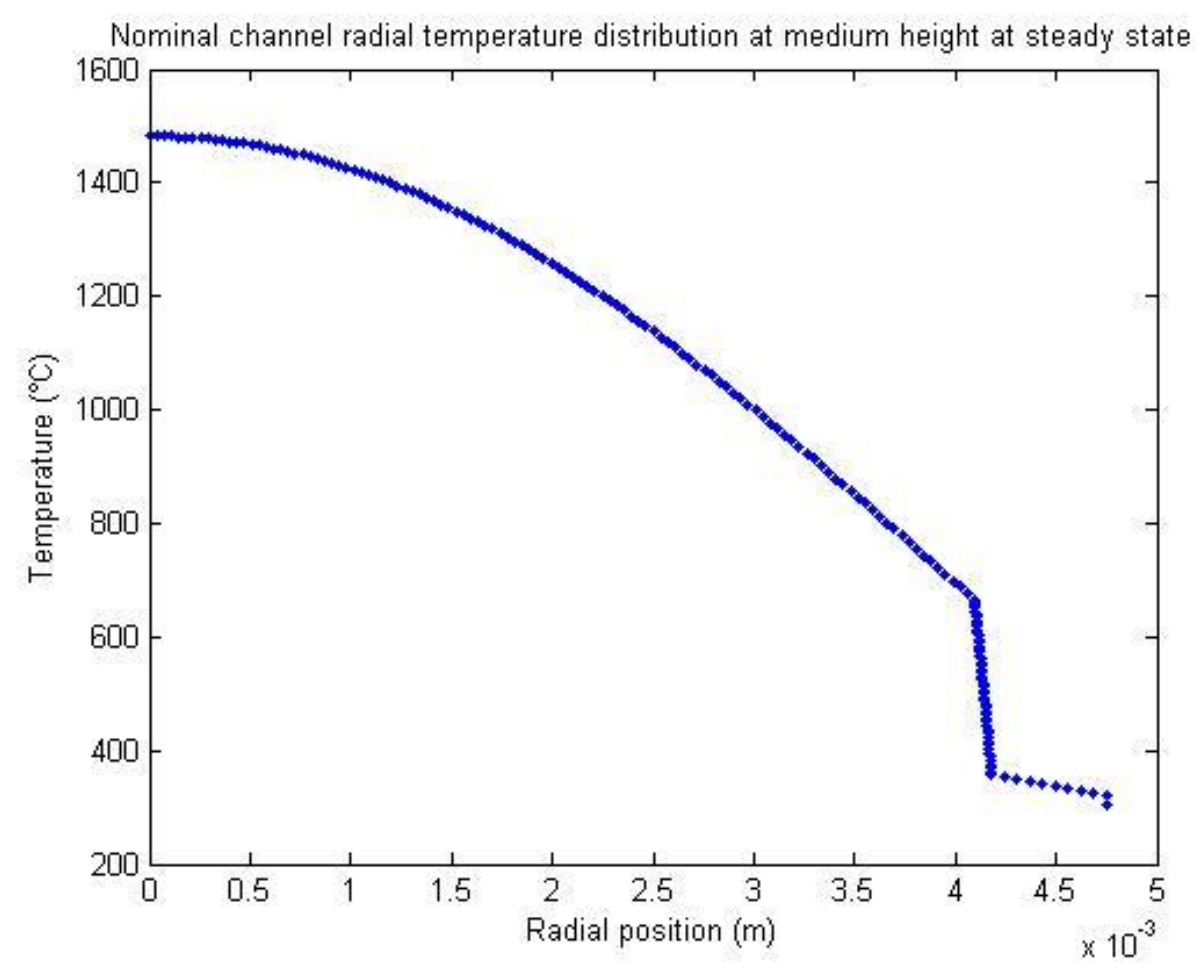

Figures 3 and 4 show the comparison between the temperature distribution obtained by numerical analysis (that considers both axial and radial heat dissipation within the fuel rod) and the analytical approach through Equations (3) and (4), that considers only radial heat dissipation inside the fuel rod.

Figure 3 presents the temperature distribution for the coolant of a AP1000's nominal channel through Equation (3) and the numerical solution. The solution obtained by Equation (3) is up to $8.14^{\circ} \mathrm{C}$ higher than the one obtained by numerical analysis.

Figure 3: Steady state coolant axial temperature distribution 


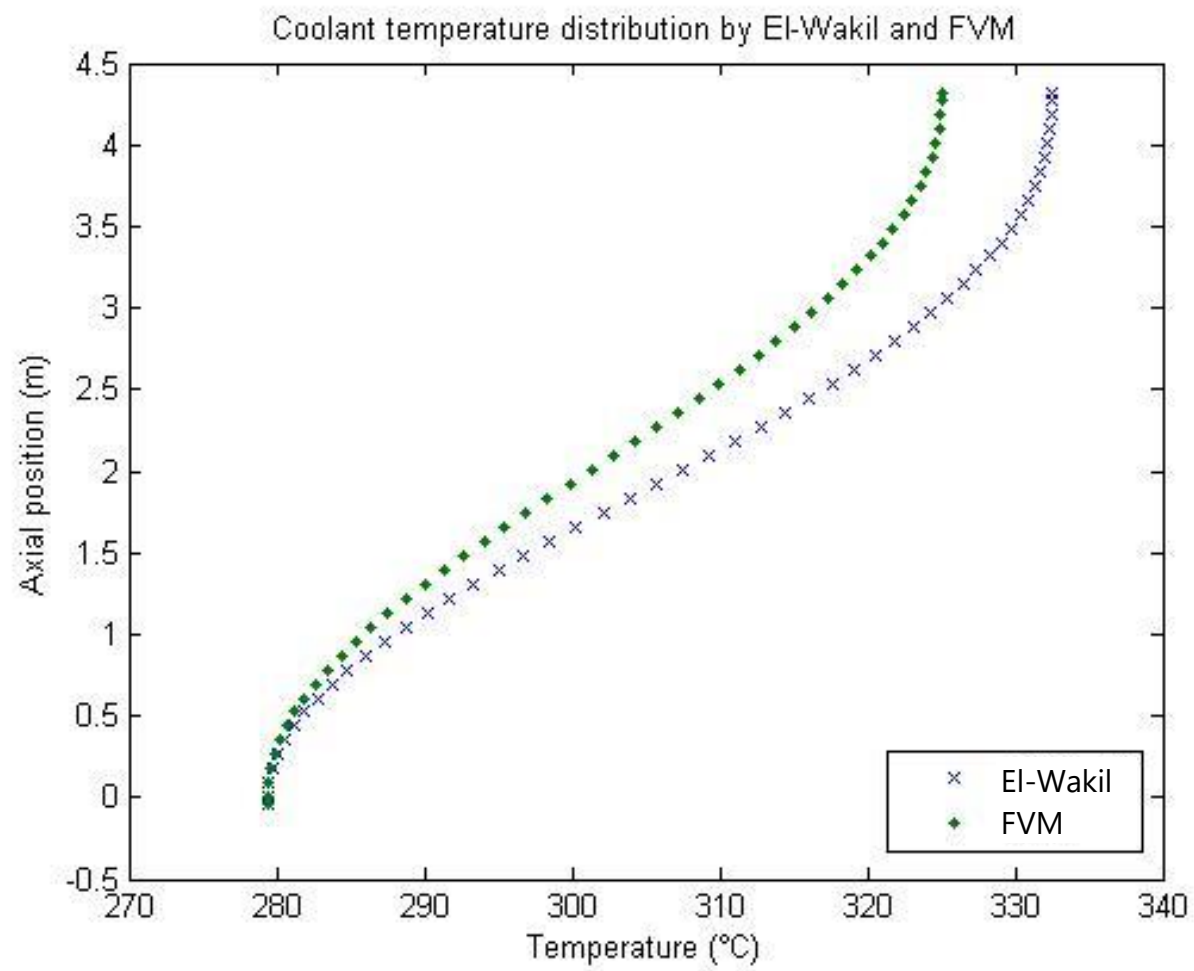

Figure 4 presents the temperature distribution at fuel rod's outer wall, by Equation (4) and FVM. The solution obtained by Equation (4) is up to $12.86^{\circ} \mathrm{C}$ higher than the one obtained by numerical analysis. 
Figure 4: Steady state outer wall's axial temperature distribution.

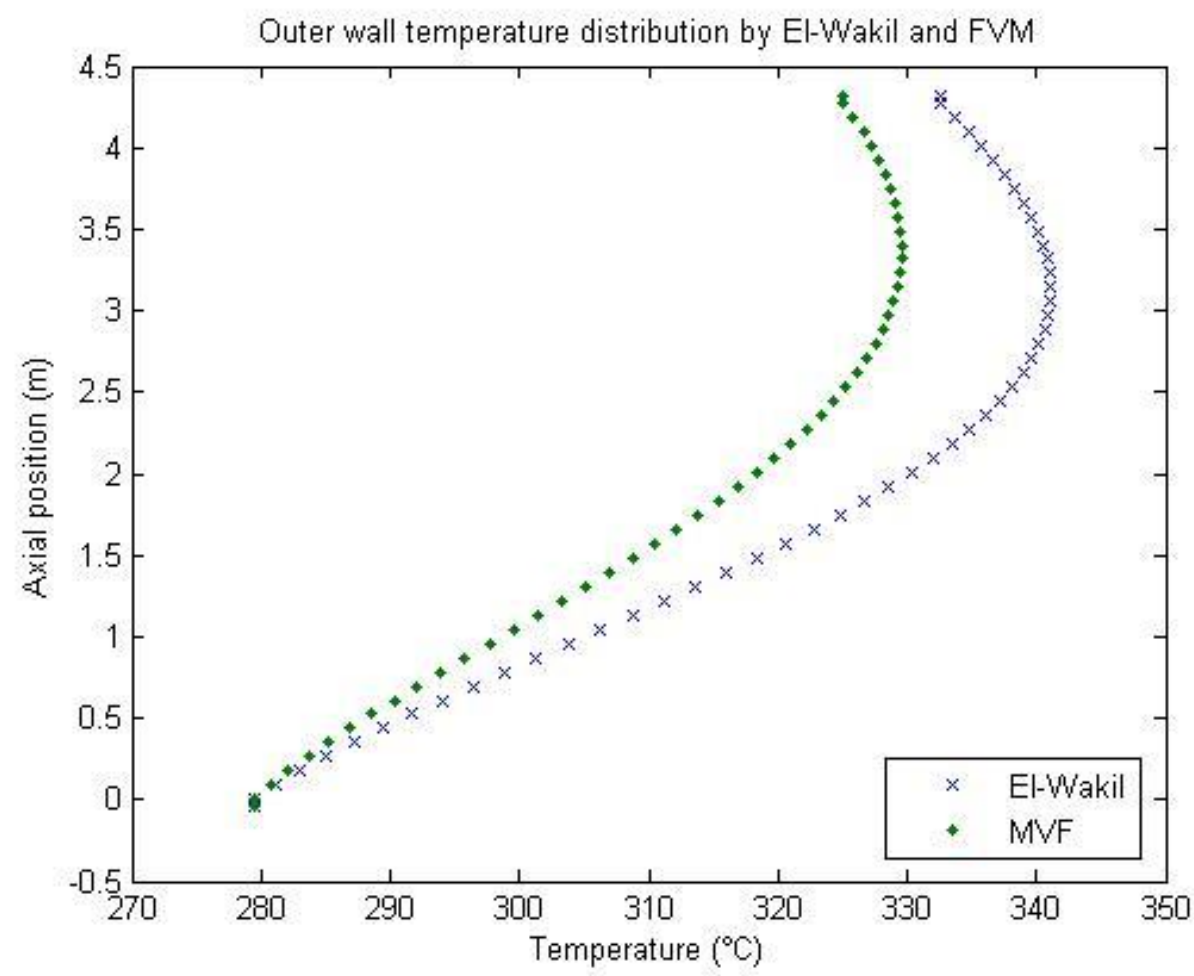

\section{TRANSIENT ANALYSIS}

The transient analysis determines the temperature distributions of a channel that was in normal operation initially (solution of steady analysis) when suffers from sudden coolant flow loss at channel's entrance (blockage in the entrance of channel), which means the same special mesh was adopted $\left(N_{z}=50, N_{f}=114, N_{g}=50, N_{c}=10\right)$. In that way, the only reason for the temperature variance is the coolant mass flow, while all other system conditions remain constant. The temperature distribution is calculated until a new steady state is reached (between two time steps, the absolute difference between each temperature is no bigger than $10^{-3}{ }^{\circ} \mathrm{C}$ ) while verifying if the safety of the channel is maintained. For this work, it was considered that the system is safe as long as no bubble formation is observed, since that would result in variation of the of phase and therefore a drastic variation of thermal properties. It is known that, until a certain degree, bubble formation does not eliminate the safety of a reactor, but, for this work, it was considered out of the safety conditions. Thus, the conditions of the coolant right near the rod's outer wall is verified to determine if 
the coolant is under saturation condition (quality lower than 0 ). Three new coolant flows were simulated: $10 \%, 20 \%$ and $30 \%$ lower than the flow before blockage. Also, the same special

\subsection{0\% BLOCKAGE}

For $10 \%$ blockage and a temporal discretization of $\Delta t=0.01 \mathrm{~s}$, the system reaches a new steady state after 128 temporal iterations. Figure 5 presents the temperature distribution at fuel rod's outer wall for the steady states before and after blockage. There is an increase up to $2.40^{\circ} \mathrm{C}$ at outer wall's temperature due to blockage, but there is no bubble formation at any point of the channel nor any instant. The temperature rise in the whole system reaches up to $3.04^{\circ} \mathrm{C}$.

Figure 5: Temperature distribution due to 10\% coolant blockage at fuel rod's outer wall.

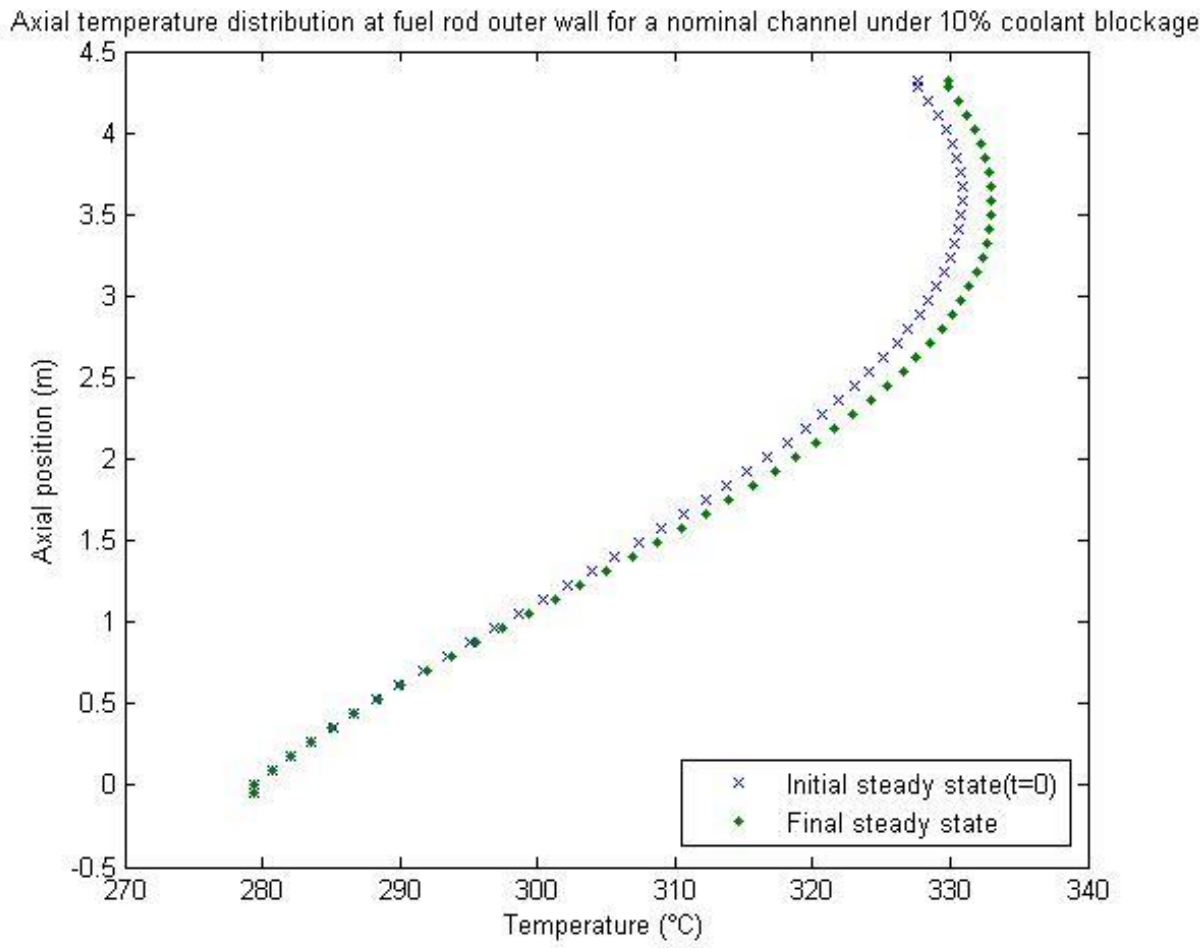


Also, even when adopting Equation (4) (that disregards the axial heat dissipation within the fuel rod) to determine outer wall's distribution, the conditions would not allow bubble formation in any position or any time, even though the temperature at outer wall would increase up to $16.75^{\circ} \mathrm{C}$.

\section{2. $20 \%$ BLOCKAGE}

For $20 \%$ blockage and a temporal discretization of $\Delta t=0.01 \mathrm{~s}$, the system reaches a new steady state after 144 temporal iterations. Figure 6 presents the temperature distribution at fuel rod's outer wall for the steady states before and after blockage. There is an increase up to $7.30^{\circ} \mathrm{C}$ at outer wall's temperature due to blockage and, again, no bubble formation during the test. The temperature rise in the whole system reaches up to $7.54^{\circ} \mathrm{C}$.

Figure 6: Temperature distribution due to $20 \%$ coolant blockage at fuel rod's outer wall.

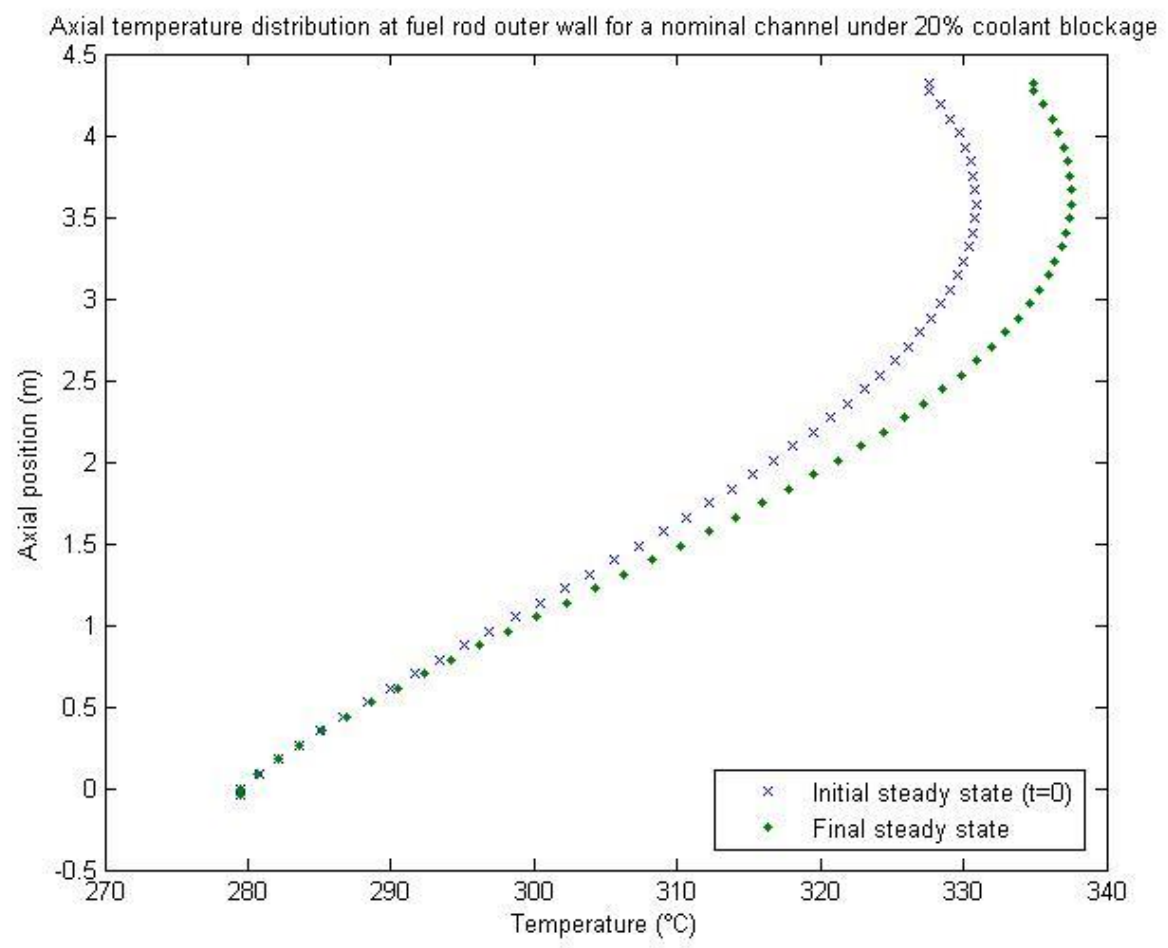


Also, when adopting Equation (4) to determine outer wall's distribution, dismissing the acknowledgement of axial heat dissipation, the temperature at outer wall could increase up to $19.96^{\circ} \mathrm{C}$. The conditions would allow bubble formation since approximately medium height in the channel, reaching an unstable state.

\section{3. $30 \%$ BLOCKAGE}

For $30 \%$ blockage and a temporal discretization of $\Delta t=0.01 s$, the system reaches a new steady state after 161 temporal iterations. Figure 7 presents the temperature distribution at fuel rod's outer wall for the steady states before and after blockage. There is an increase up to $13.33^{\circ} \mathrm{C}$ at outer wall's temperature due to blockage, and no bubble formation anywhere or anytime.

Figure 7: Temperature distribution due to 30\% coolant blockage at fuel rod's outer wall.

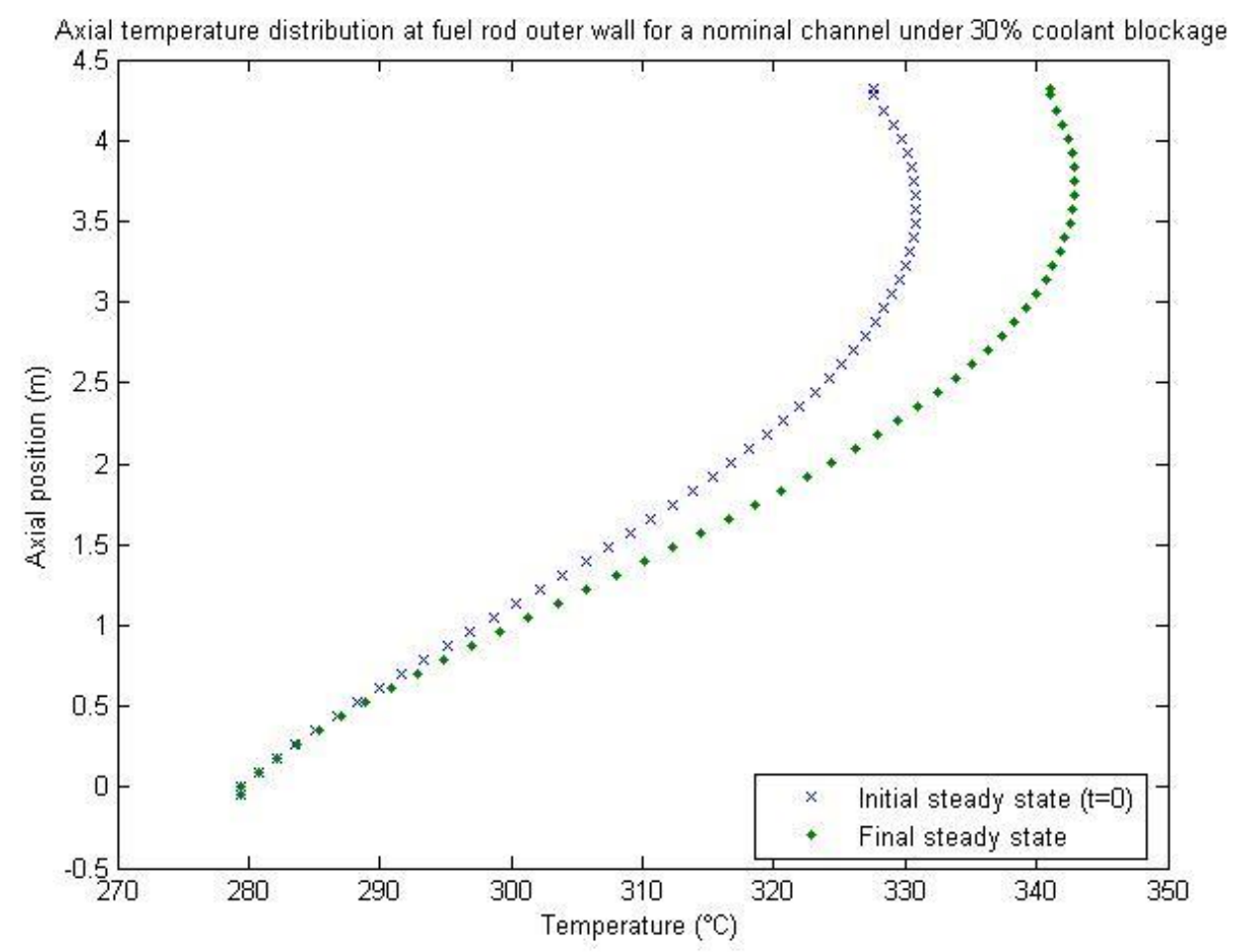


The temperature rise in the whole system reaches up to $13.43^{\circ} \mathrm{C}$. Furthermore, when adopting Equation (4) to determine outer wall's distribution, the conditions would allow bubble formation from approximately medium height in the channel $(z=2,269 m)$. By dismissing the acknowledgement of axial heat dissipation, the temperature at outer wall could increase up to $23.58^{\circ} \mathrm{C}$.

\section{CONCLUSION}

After the three transient analysis, none of the blockages would cause bubble formation (nowhere in the wall reaches a condition with quality lower than 0 ). The blockages could cause a temperature increase higher than $13^{\circ} \mathrm{C}$ in the wall and the entire channel's temperature distributions. Once again, the dismissal of axial heat dissipation through fuel rod's material (de adoption of Equation (4)) would cause an overestimation of those values up to $10^{\circ} \mathrm{C}$ at rod's outer wall temperature, which would allow bubble formation from about medium height in the evidenced channel, and system instabilities for blockages higher than $10 \%$ of mass flow of coolant at channel entrance.

For greater blockages than 30\%, the system couldn't reach convergence. Since no model for bubble formation was adopted, when the saturation was near, coolant properties varied changing abruptly system's behavior that was not able to correct itself to expected behavior. That resulted in inconclusive results since the meaning was not that the system would not reach a new stable state, or that it actually resulted in a real instability, since it is known that some bubble formation does not represents danger in a reactor. That only means that, due to the lack of the developed code, that is not ready to deal with biphasic states, when the system started to present bubbles, the results were not reliable.

Finally, the code developed considered only the fluid temperature as a factor of safety, but much more should have been considered. The temperatures grow as fuel rod center is approached and those temperature should be monitored to guarantee that all materials limits are also respected. 


\section{ACKNOWLEDGMENT}

The authors thank the parcial support of CNPq (Conselho Nacional de Desenvolvimento Científico e Tecnológico) and colleagues from Federal University of Pernambuco for all the help.

\section{REFERENCES}

[1] MATZIE, R. A. AP1000 will meet the challenges of near-term deployment. Nuclear Engineering and Design, v. 238, p. 1856-1862, 2008.

[2] SCHULZ, T. L. Westinghouse AP1000 advanced passive plant. Nuclear Engineering and Design, v. 236, p. 1547-1557, 2006.

[3] YU, H.; TIAN, W.; YANG, Z.; SU, G. H.; QIU, S. Development of Fuel ROd Behavior Analysis code (FROBA) and its application to AP1000. Annals of Nuclear Energy, v. 50, p. 8-17, 2012. [4] LAMARSH, J. R. Introduction to nuclear reaction theory, Addison Wesley Publishing Company, 1966.

[5] VERSTEEG, H. K.; MALALASEKERA, W., An Introduction to Computational Fluid Dynamics: The Finite Volume Method, Pearson Education, 2007.

[6] EL-WAKIL, M. M., Nuclear Heat Transport, Scraton: International Textbook Company, 1971.

[7] KIM, H. T.; RHEE, B. W.; PARK, J. H. Application of the finite volume method to the radial conduction model of the CATHENA code. Annals of Nuclear Energy, v. 33, p. 924-931, 2006.

[8] SILVA, M. A. B.; NARAIN, R. A quantitative estimate on the heat transfer in cylindrical fuel rods to account for flux depression inside fuel. Progress in Nuclear Energy, v. 69, p. 29-34, 2013. [9] AINSCOUGH, J. B. Gap conductance in Zircaloy-clad LWR fuel rods. United Kingdon Atomic Energy Authority, 1982.

[10] IAEA - International Atomic Energy Agency. Thermophysical Properties Database of Materials for Light Water Reactors and Heavy Water Reactors, Vienna: IAEA, 2006, 397p. 
[11] Westinghouse Electric Company - Westinghouse AP1000 Design Control Document Rev. 19 Available at: <https://www.nrc.gov/docs/ML1117/ML11171A500.html>. Last accessed: 14 Nov. 2017.

[12] LUSCHER, W. G.; GEELHOOD, K. J. Material Property Correlations: Comparisons between FRAPCON-4.0, FRAPTRAN 2.0, and MATPRO. U.S. Nuclear Regulatory Commission: Office of Nuclear Regulatory Research, 2014.

[13] WHITMARSH, C. L. Review of Zircaloy-2 and Zircaloy-4 Properties Relevant to N.S. Savannah Reactor Design. No. ORNL-3281, Oak Ridge: Oak Ridge National Laboratory, Atomic Energy Commission, 1962.

[14] NIST - National Institute of Standards and Technology, NIST Reference Fluid Thermodynamic and Transport Properties Database (REFPROP): Version 9.1 User's Guide, National Institute of Standards and Technology, 2013.

[15] COSTA, R. Y. N. Análise térmica bidimensional de uma barra de combustível nuclear pelo método dos volumes finitos sob fluxo neutrônico variável. MS thesis. Universidade Federal de Pernambuco, 2017. 